\title{
Full and Partial Amidation of Poly(methyl acrylate) as basis for functional polyacrylamide (co)polymers
}

\author{
Joachim F. R. Van Guyse, Jente Verjans, Stef Vandewalle, Kevin De Bruycker ${ }^{\dagger}$, Filip E. Du \\ Prez, Richard Hoogenboom*
}

\section{Experimental}
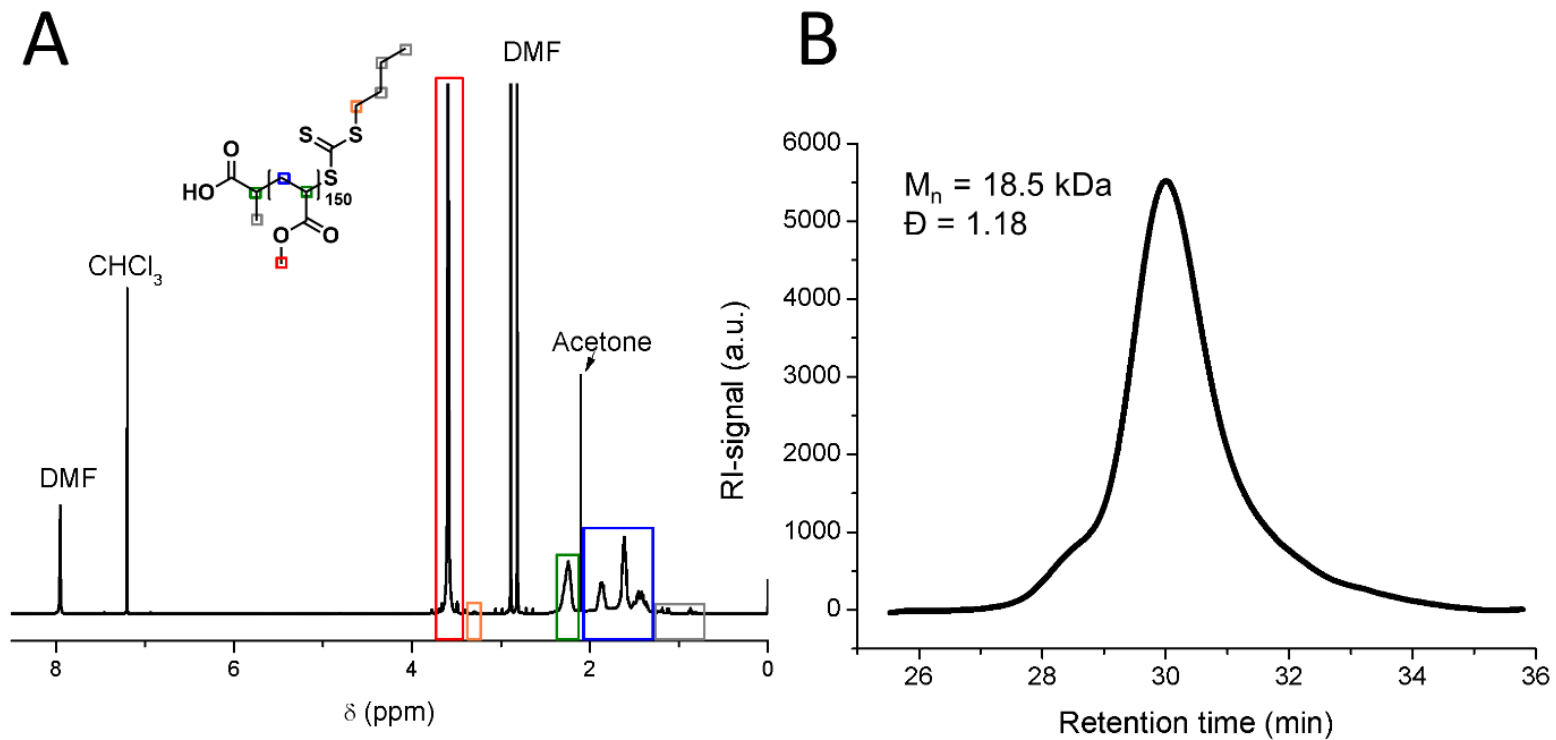

Figure S1 A) ${ }^{1} H$ NMR spectrum of PMA with the respective annotated proton annotations. B) Size-exclusion chromatogram (refractive index (RI)-trace) of PMA obtained with DMA with $\mathrm{LiCl}(50 \mathrm{mM})$ as eluent, number average molecular weight $(\mathrm{Mn})$ and dispersity $(\Theta)$ are given relative to PMMA standards.

\section{Calculation of conversion}

The calculation of conversion was performed by utilizing the signals of the olefin groups of allylamine (signal at 6 ppm was utilized) and cyclohexenylethylamine (signals at $5.5 \mathrm{ppm}$ ), which integrate for 1 proton, as well as utilizing the signal of the ester present at $3.6 \mathrm{ppm}$, integrating for 3 protons of the methyl ester. The calculation was done as following:

$$
\frac{\text { Integral olefin }}{\left(\text { integral olefin }+\frac{\text { integral ester }}{3}\right)}=\text { conversion }
$$

For benzyl amine, the $\mathrm{CH} 2$ protons of the benzyl group were utilized (sum of integrals of signals at 4.9 and $4.4 \mathrm{ppm}$ ) which integrate for 2 protons, and the signal of the ester present at $3.6 \mathrm{ppm}$, integrating for 3 protons of the methyl ester. The calculation was done as following:

$$
\frac{\frac{\text { integral benzylamide }}{2}}{\left(\frac{\text { integral benzylamide }}{2}+\frac{\text { integral ester }}{3}\right)}=\text { conversion }
$$


For the equimolar mixture of primary amines, the relative amide content of the respective amines was determined in a similar manner, the total conversion could be determined by the sum of the individual conversions of each amine. Finally, for pyrrolidine and $\mathrm{N}, \mathrm{N}$-diethylamine, a different approach was utilized due to the lack of well resolved olefin protons. For this purpose, the $\mathrm{CH}$ signal from the ploymer backbone (2.8- $2.1 \mathrm{ppm}$ ) which remains constant during the modification reaction, was compared to the diminishing signal of the ester groups of the methyl acrylate groups ( $3.6 \mathrm{ppm}$ ). The calculation was done as following:

$$
\frac{\frac{\text { integral ester }}{3}}{(\text { integral } \text { CH backbone })}=\text { conversion }
$$

\section{Variation of equivalents of amine to ester}

PMA (200 mg, 1 equivalent of ester groups, $2.3 \mathrm{~mol}$ ), TBD (138 mg, 0.5 eq, $1.15 \mathrm{~mol}$ ) and varying equivalents $(0.5,2,4,6 \mathrm{eq}$ relative to ester groups) of allylamine were dissolved in an appropriate amount of $A C N$, so that the total volume of the mixture would be $10 \mathrm{~mL}$. Next, the mixture was heated to $70^{\circ} \mathrm{C}$ and left to react, while $1 \mathrm{~mL}$ samples were taken at regular intervals. These samples were precipitated in $10 \mathrm{~mL}$ cold diethyl ether in a $15 \mathrm{~mL}$ centrifuge tube. The resulting opaque mixture was centrifuged at 7500 revolutions per minute (rpm), after which the solvent was decanted and the kinetic sample was dried overnight in a vacuum oven at $50^{\circ} \mathrm{C}$. These samples were then subjected to ${ }^{1} \mathrm{H}$ NMR and SEC analysis. In ${ }^{1} \mathrm{H}$ NMR the conversion was determined by integrating the same interval corresponding to the methyl ester signal and the formed amide signal, dividing those integrals by the corresponding protons they represent and taking the ratio of those values.

\section{Variation of equivalents of TBD to ester and temperature}

PMA (200 mg, 1 equivalent of ester groups, $2.3 \mathrm{~mol}$ ), TBD ( $276 \mathrm{mg}, 1 \mathrm{eq}, 2.3 \mathrm{~mol}$ ) and ( 6 eq relative to ester groups) allylamine were dissolved in an appropriate amount of ACN, so that the total volume of the mixture would be $10 \mathrm{~mL}$. Next, the mixture was heated to $70^{\circ} \mathrm{C}$ and left to react, while $1 \mathrm{~mL}$ samples were taken at regular intervals. These samples were precipitated in $10 \mathrm{~mL}$ cold diethyl ether in a $15 \mathrm{~mL}$ centrifuge tube. The resulting opaque mixture was centrifuged at 7500 revolutions per minute (rpm), after which the solvent was decanted and the kinetic sample was dried overnight in a vacuum oven at $50^{\circ} \mathrm{C}$. These samples were then subjected to ${ }^{1} \mathrm{H}$ NMR spectroscopy. In the ${ }^{1} \mathrm{H}$ NMR spectra the conversion was determined by integrating the same interval corresponding to the methyl ester signal and the formed amide signal, dividing those integrals by the corresponding protons they represent and taking the ratio of those values.

\section{Variation of total concentration}

PMA (200 mg, 1 equivalent of ester groups, $2.3 \mathrm{~mol}$ ), TBD (138 mg, $0.5 \mathrm{eq}, 2.3 \mathrm{~mol}$ ) and ( 6 eq relative to ester groups) allylamine were dissolved in an appropriate amount of $A C N$, so that the total volume of the mixture would be $5 \mathrm{~mL}$. Next, the mixture was heated to $70^{\circ} \mathrm{C}$ and left to react, while $1 \mathrm{~mL}$ samples were taken at regular intervals. These samples were precipitated in $10 \mathrm{~mL}$ cold diethyl ether in a $15 \mathrm{~mL}$ centrifuge tube. The resulting opaque mixture was centrifuged at 7500 revolutions per minute (rpm), after which the solvent was decanted and the kinetic sample was dried overnight in a vacuum oven at $50^{\circ} \mathrm{C}$. These samples were then subjected to ${ }^{1} \mathrm{H}$ NMR spectroscopy. The conversion was determined by integrating the same interval corresponding to the methyl ester signal and the formed amide signal in the ${ }^{1} \mathrm{H}$ NMR spectra, dividing those integrals by the corresponding protons they represent and taking the ratio of those values. 
A Amount of amide: $\square 6$ eq amine $\ominus 4$ eq amine $\Delta 2$ eq amine $\downarrow 0.5$ eq amine
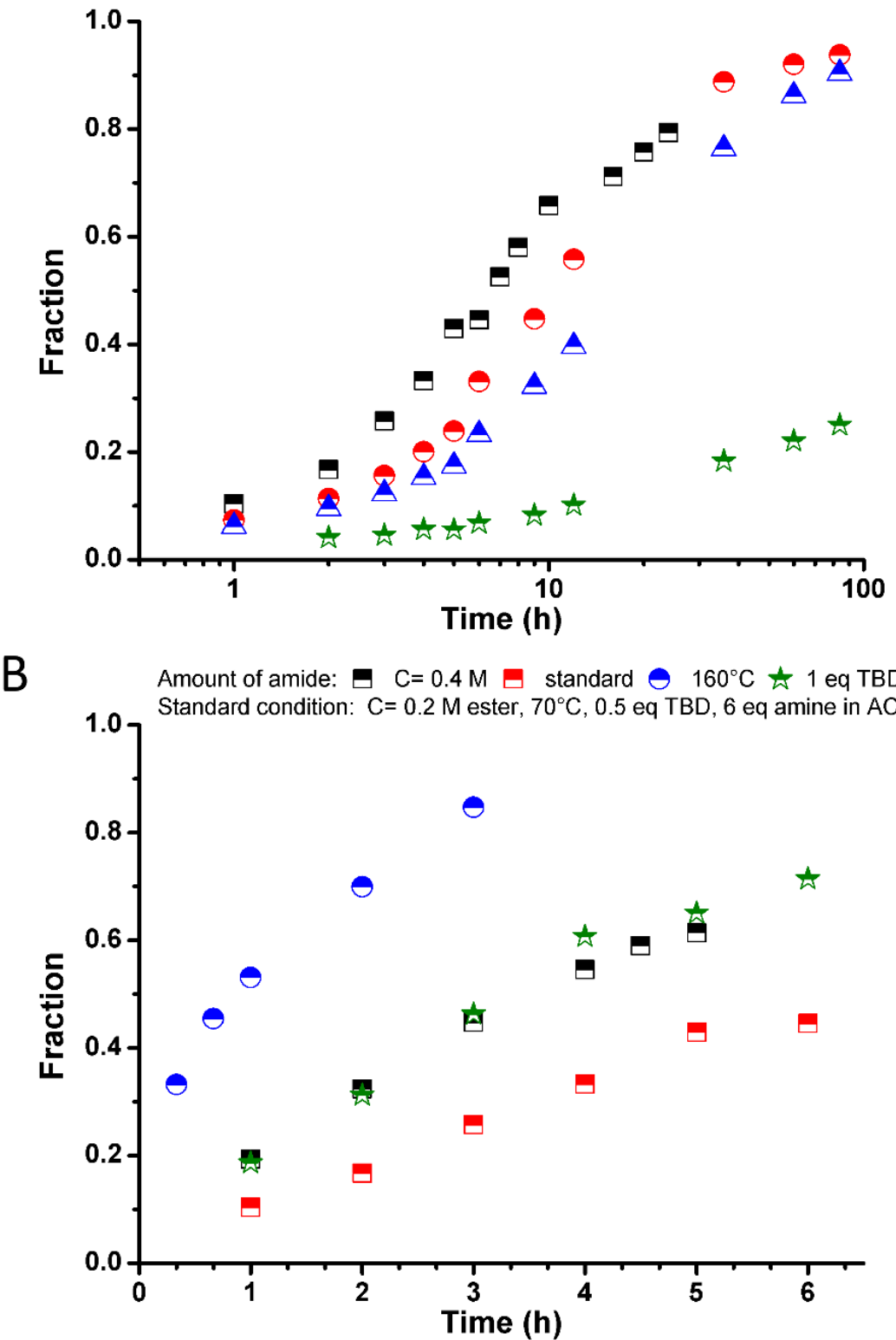

Figure S2: A) Kinetic investigation on different parameters of the amidation reaction with variation of equivalents of amine relative to ester with a logarithmic $X$-axis for graphical purposes and $B$ ) variation of ester concentration, temperature and equivalents of catalyst versus standard condition, only the specific variation versus the standard condition is listed in the legend.

Table 1: pKa values of the utilized amines for the kinetic investigation of the TBD-catalyzed amidation.

\begin{tabular}{|l|l|}
\hline Amine & pKa of conjugate acid \\
\hline Benzylamine $^{1}$ & 9.33 \\
\hline Allylamine $^{2}$ & 9.7 \\
\hline $\begin{array}{l}\text { Cyclohexenylethyl amine } \\
\text { (propylamine) }^{1}\end{array}$ & Not reported (10.71) \\
\hline$N, N$-diethylamine $^{1}$ & 10.98 \\
\hline Pyrrolidine $^{1}$ & 11.3 \\
\hline
\end{tabular}


Additional characterization data:

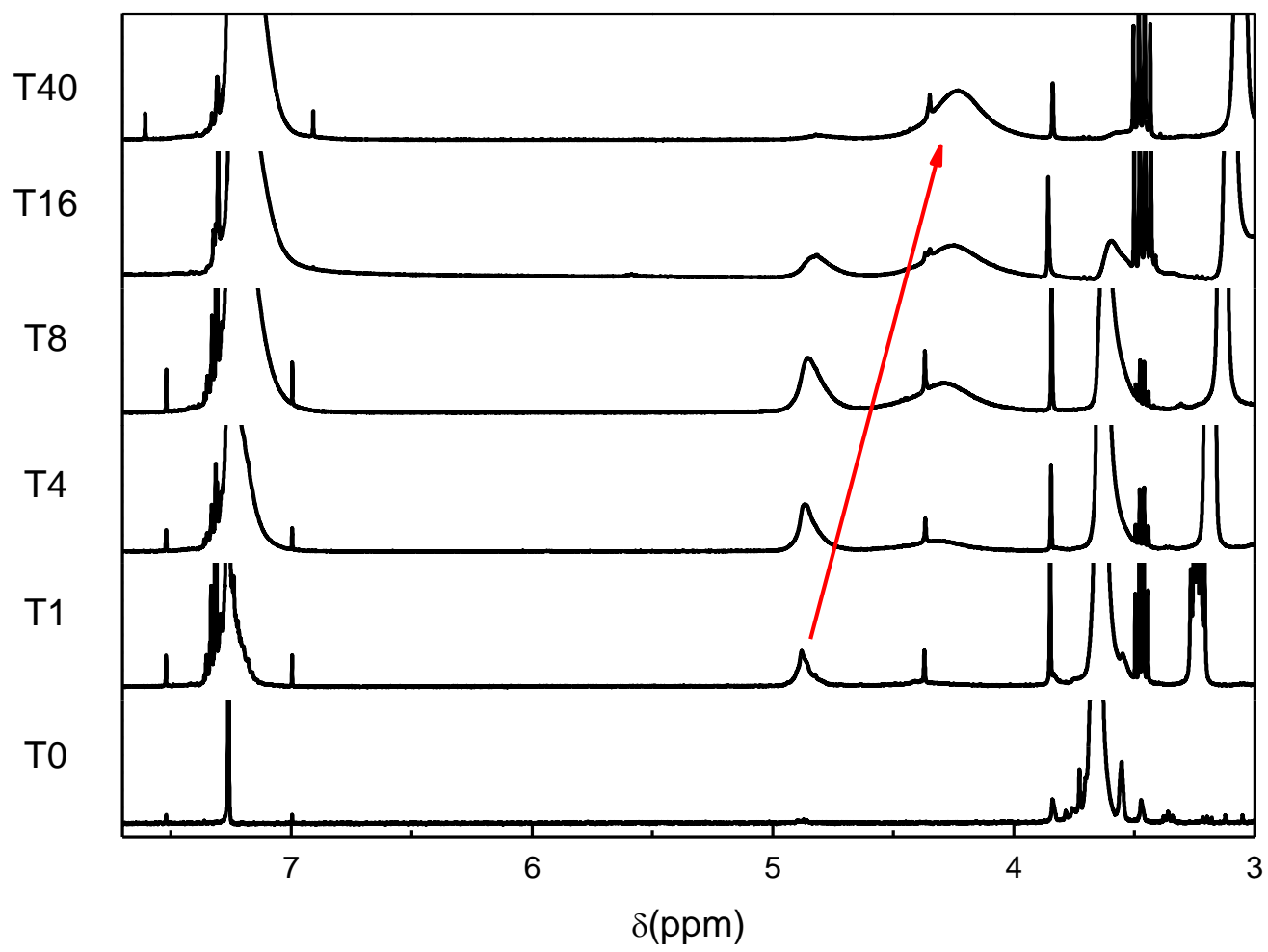

Figure S3: Stacked ${ }^{1} H$ NMR spectra of different time points in the amidation of PMA with benzylamine. The arrow indicates a change in relative intensity between the two peaks.

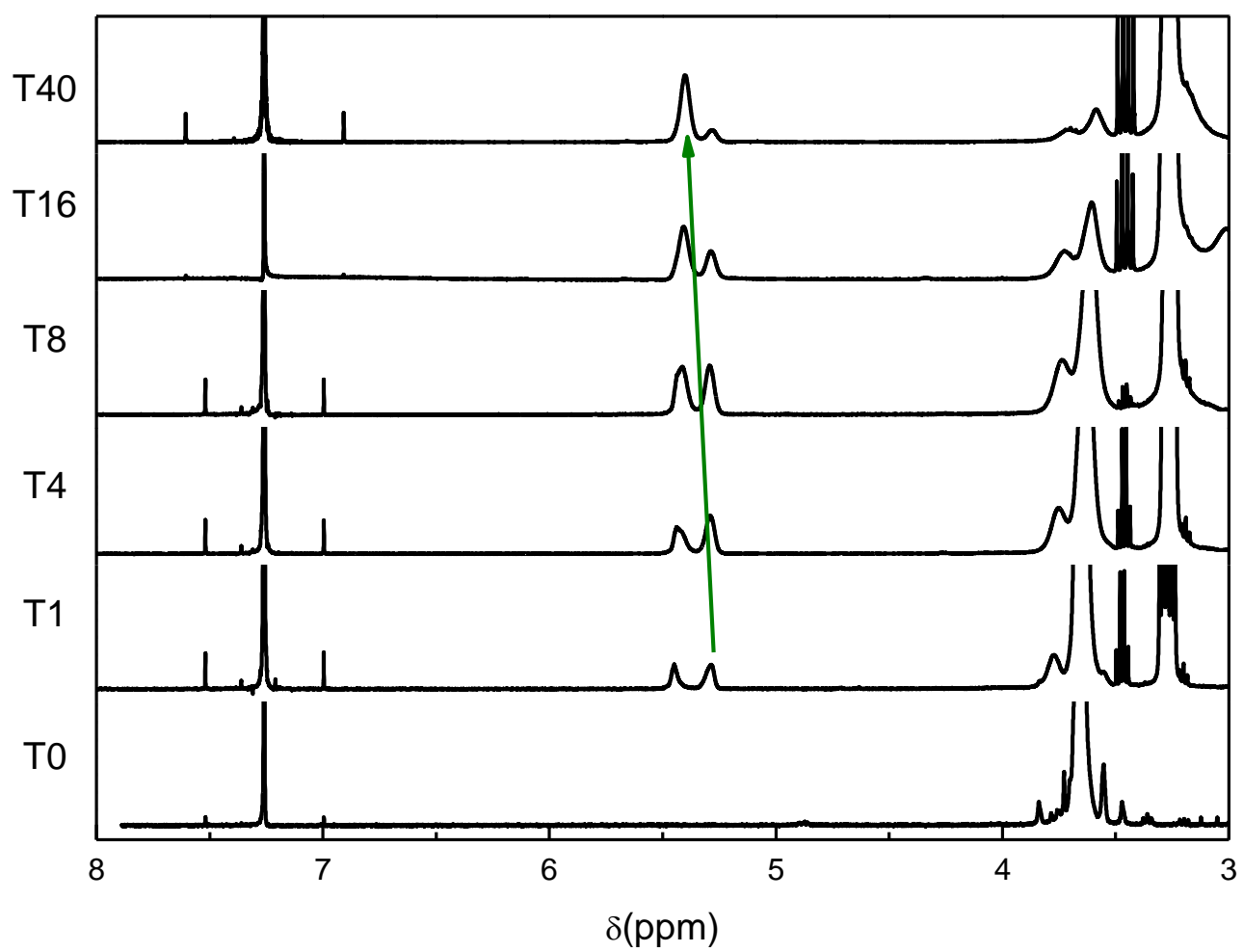

Figure S4: Stacked ${ }^{1} H$ NMR spectra of different time points in the amidation of PMA with cyclohexenylethylamine. The arrow indicates change in relative intensity between the two peaks. 


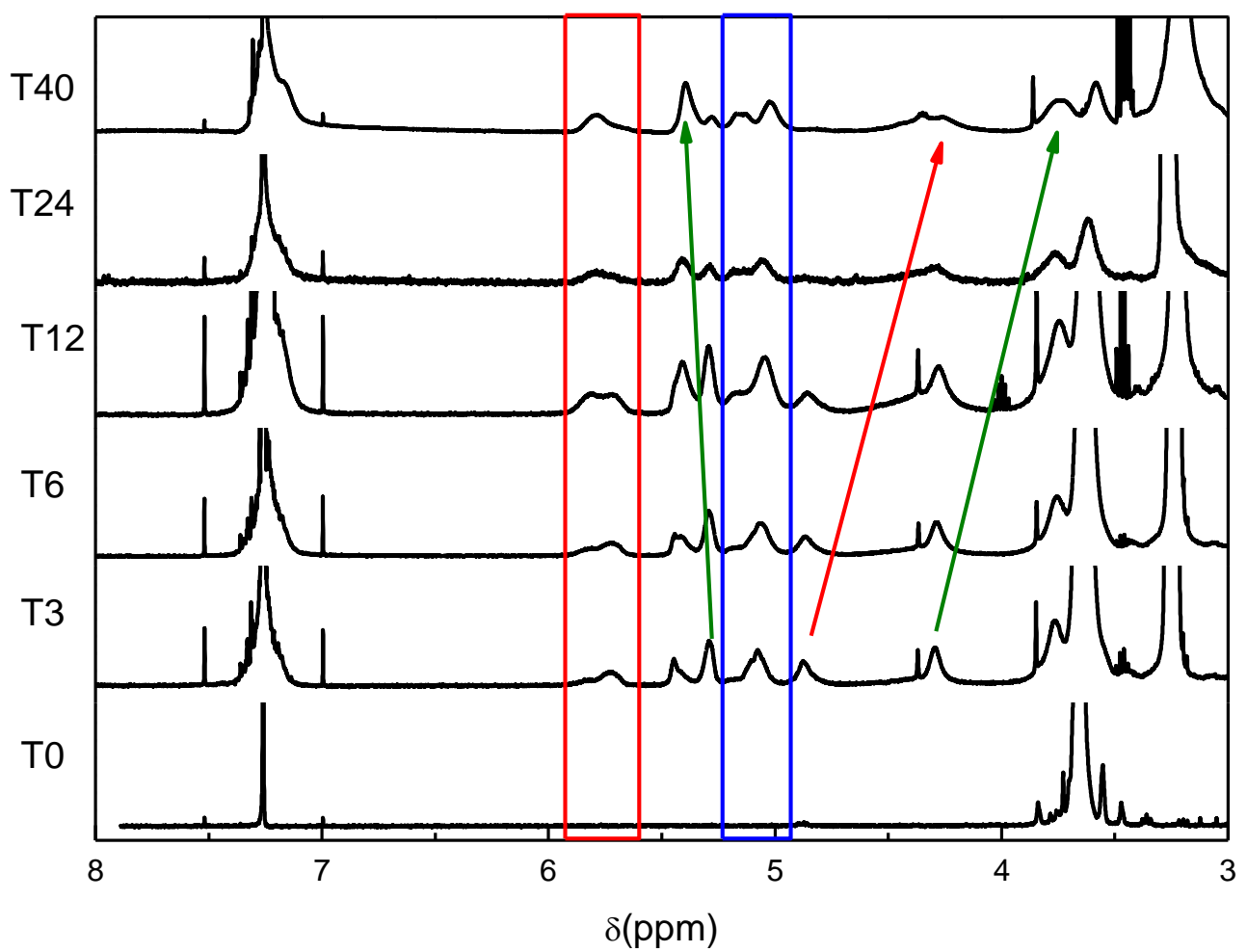

Figure S5: Stacked ${ }^{1} \mathrm{H}$ NMR spectra of different time points in the amidation of PMA with an equimolar mixture of allylamine, benzylamine and cyclohexenylethylamine. The arrow indicates change in relative intensity between the two peaks and frames indicate a change in peak shape.

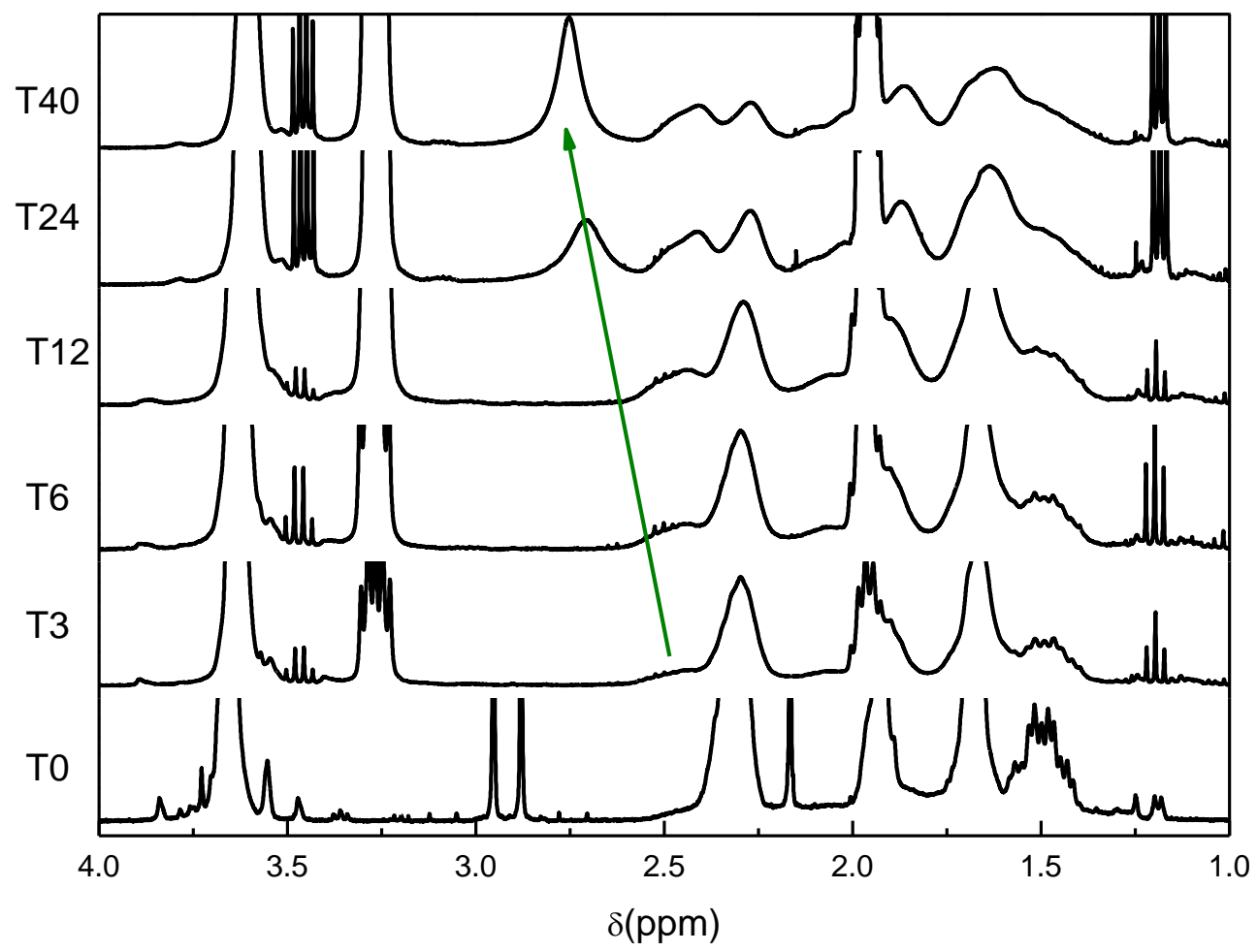

Figure S6: Stacked ${ }^{1} \mathrm{H}$ NMR spectra of different time points in the amidation of PMA with $\mathrm{N}, \mathrm{N}$-diethylamine. The arrow indicates change in relative intensity between the two peaks. 


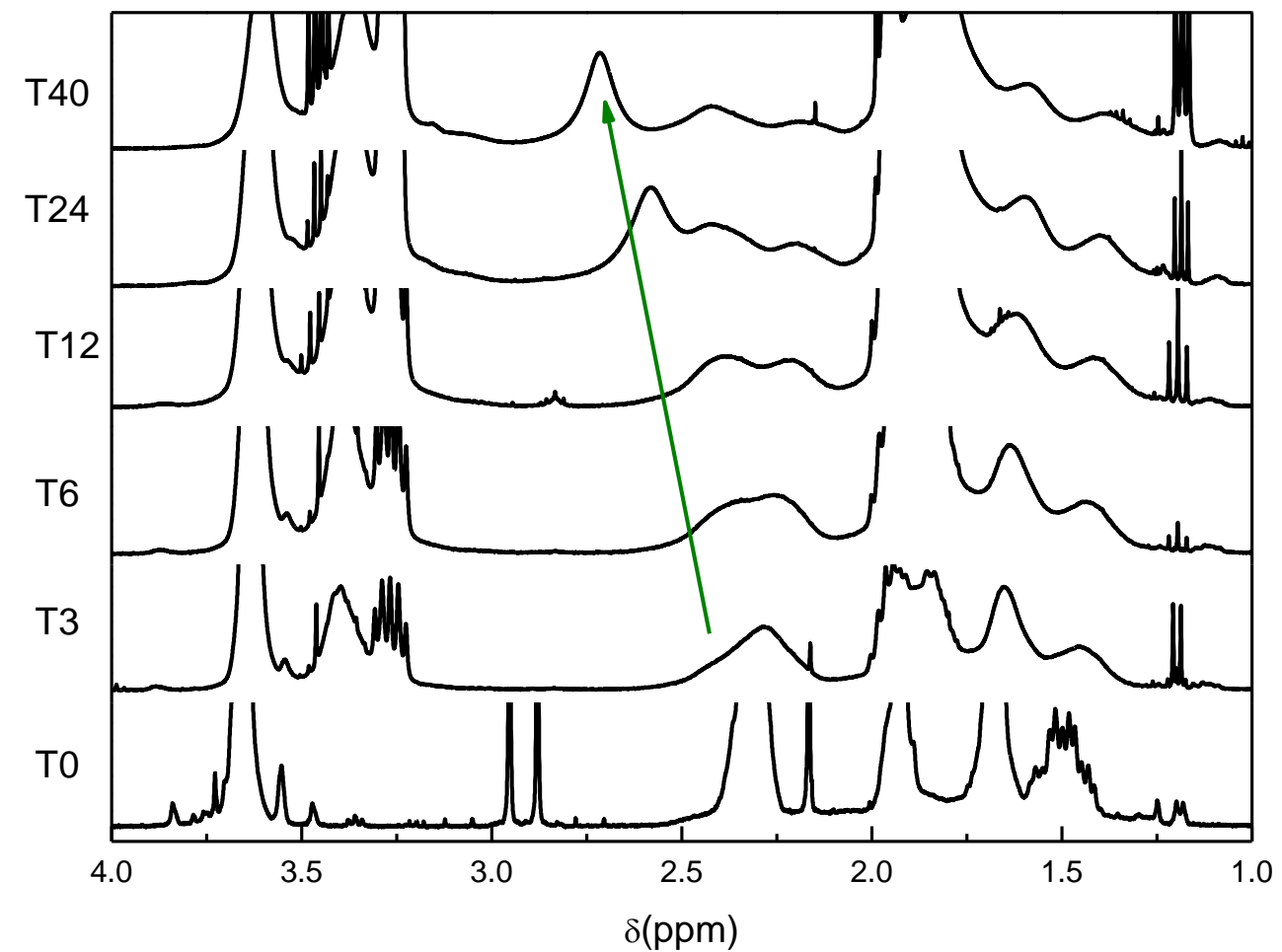

Figure S7: Stacked ${ }^{1} \mathrm{H}$ NMR spectra of different time points in the amidation of PMA with pyrrolidine. The arrow indicates change in relative intensity between the two peaks. 


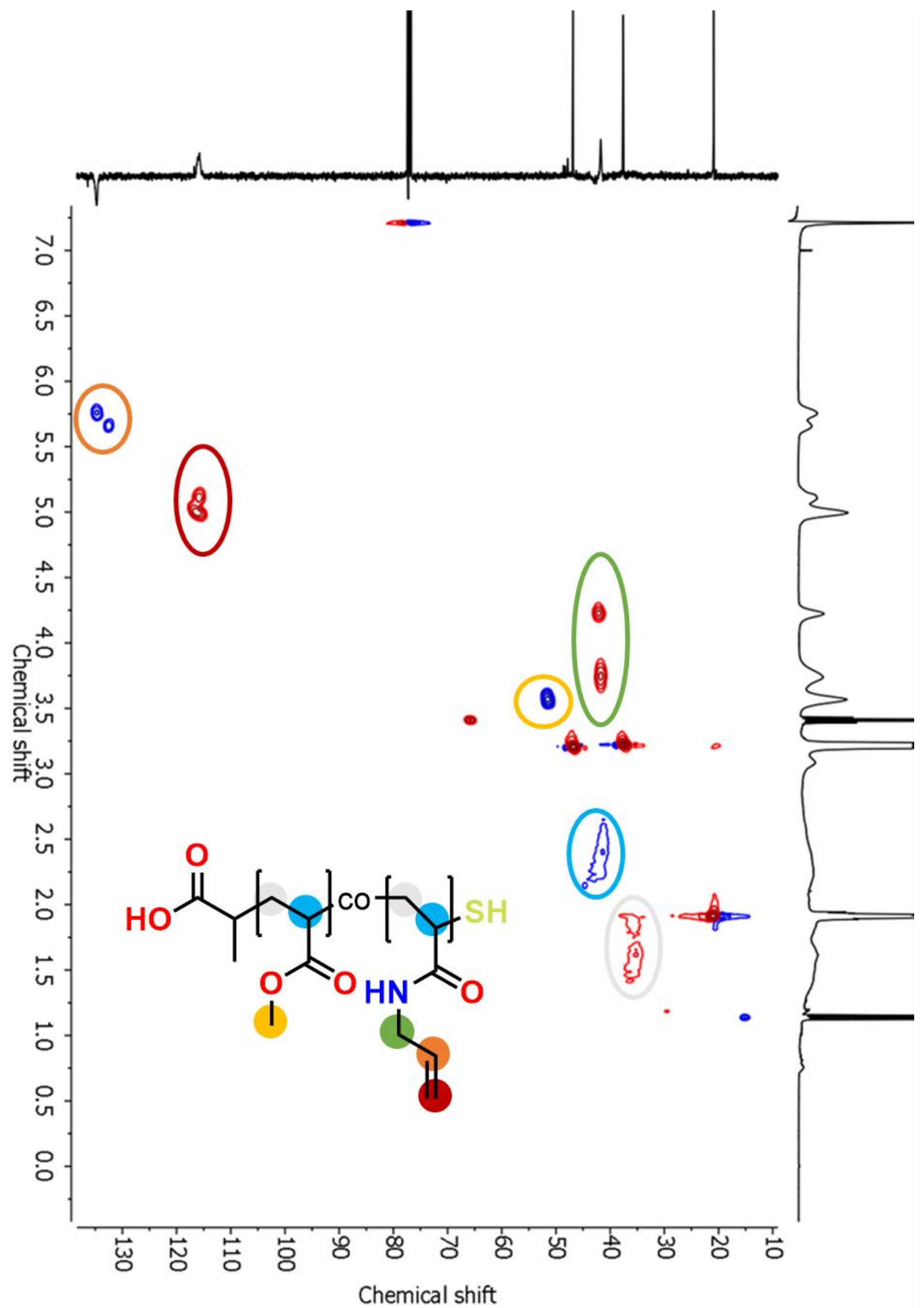

Figure S8: HSQC spectrum of $P(M A-c o-A l l y \mid A m)$ after 16 hours of reaction time. 


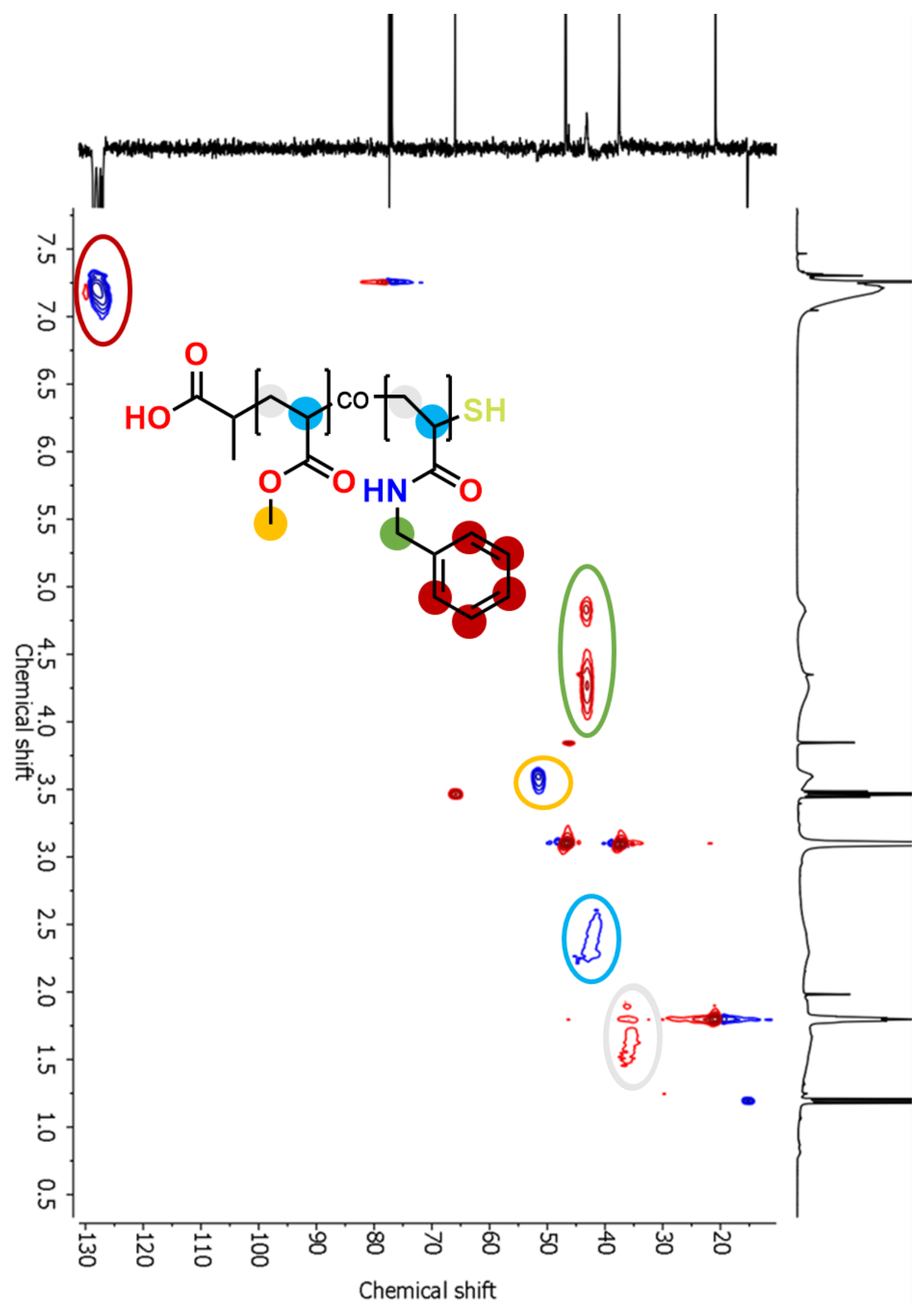

Figure S9: HSQC spectrum of $P(M A-c o-B z A m)$ after 16 hours of reaction time. 


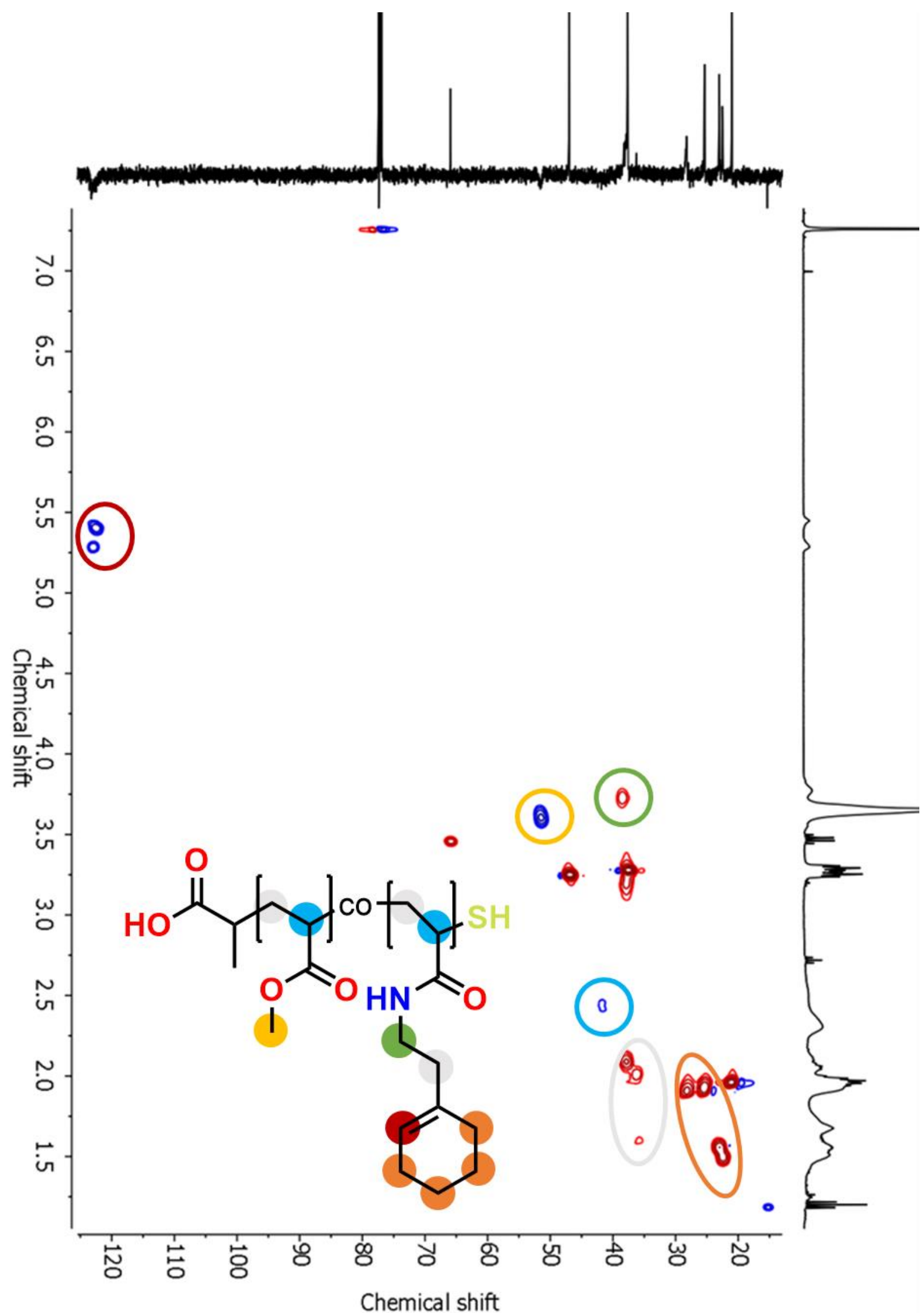

Figure S10: HSQC spectrum of $P(M A-c 0-c y c l o h e x e n y l e t h y I A m)$ after 16 hours of reaction time. 


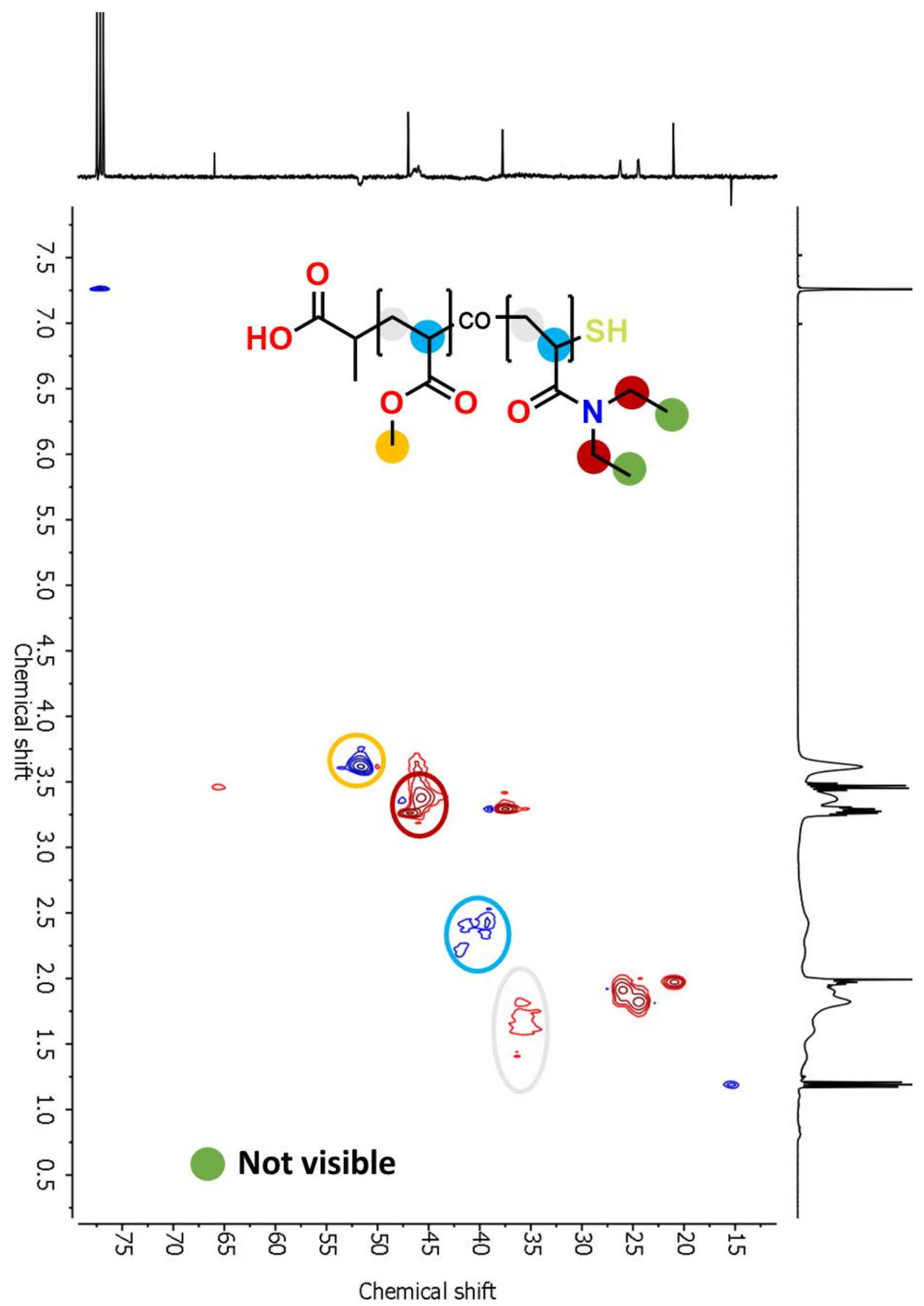

Figure S11: HSQC spectrum of $P(M A-c o-N, N$-diethylAm) after 16 hours of reaction time. 


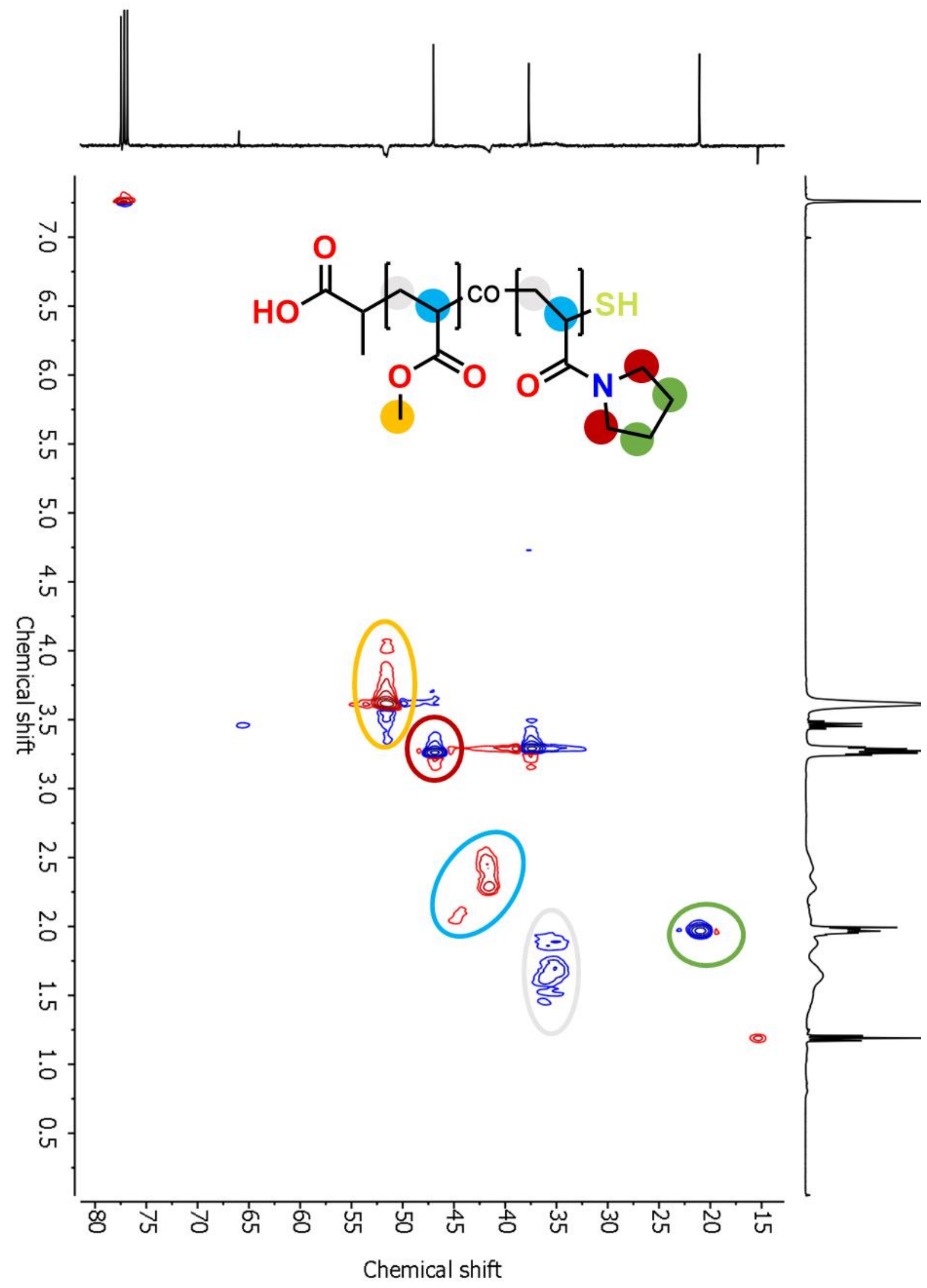

Figure S12: HSQC spectrum of $P(M A-c o-p y r r o l i d i n e A m)$ after 16 hours of reaction time. 

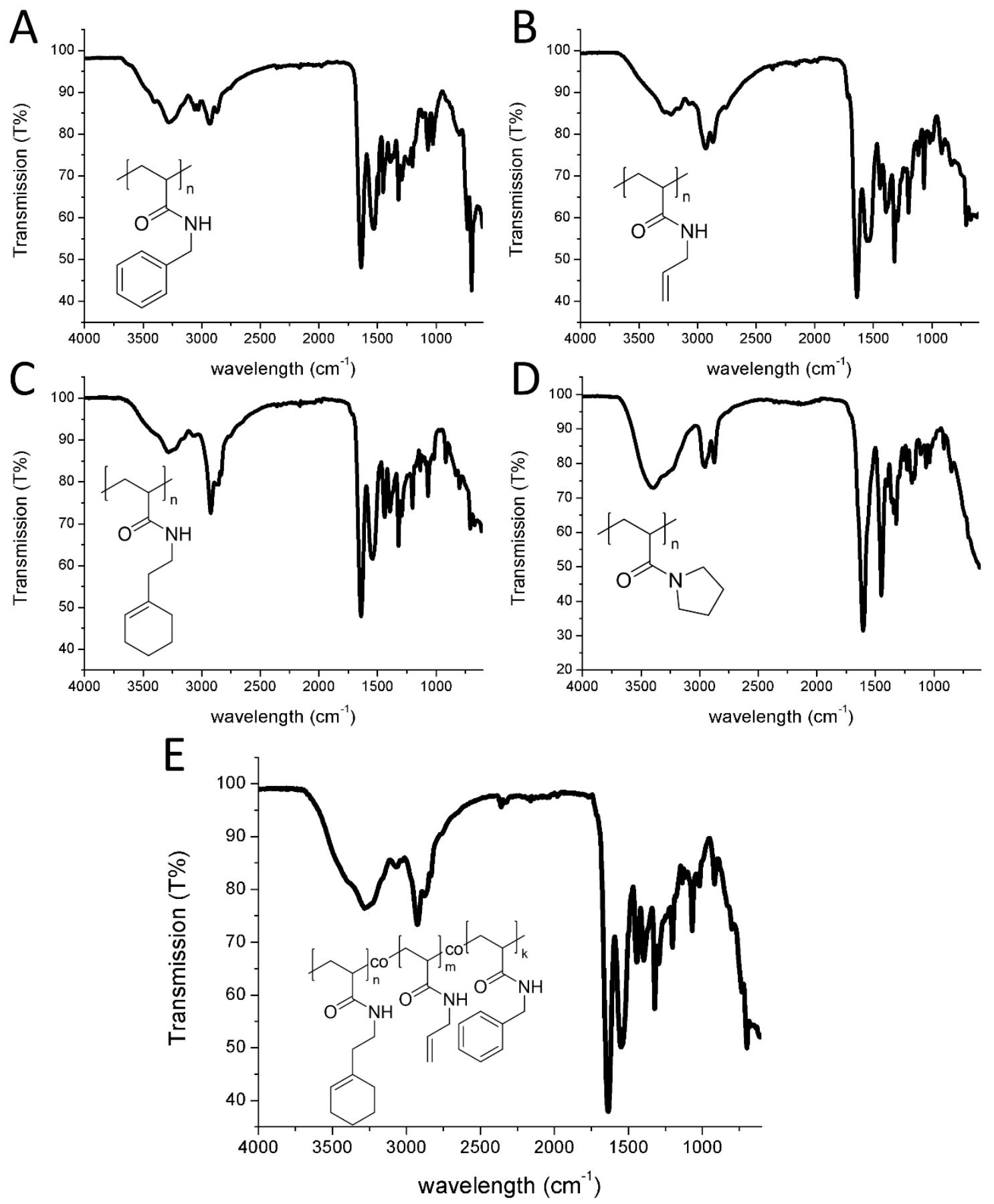

Figure S13: FT-IR-spectra of (quasi-)completely modified PMA with A) benzylamine, B) allylamine, C) cyclohexenylethylamine, $D)$ pyrrolidine and $E$ ) the equimolar mixture of the aforementioned primary amines. 
A

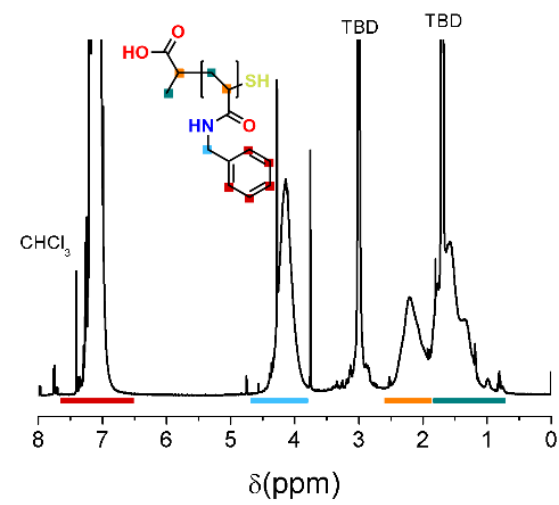

C

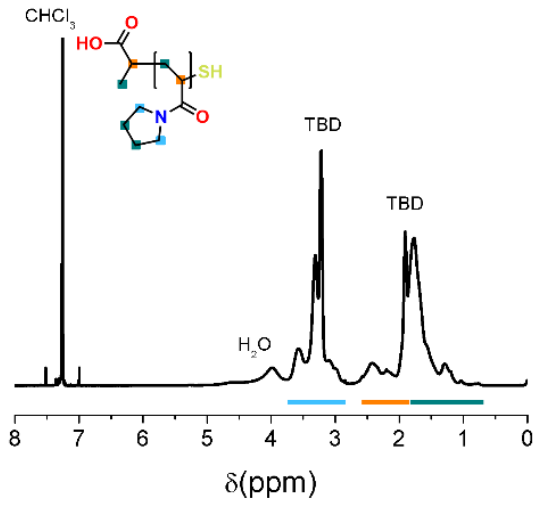

$E$

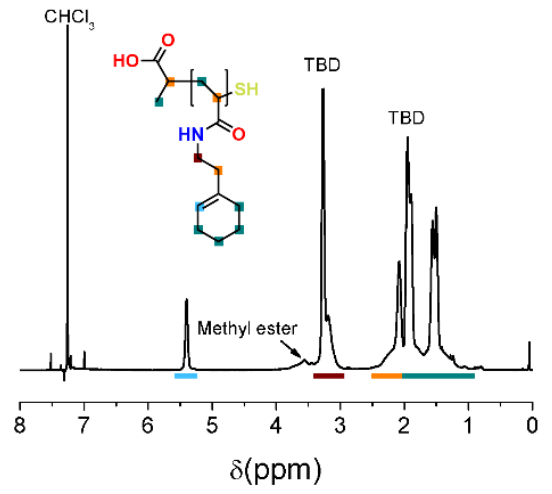

B

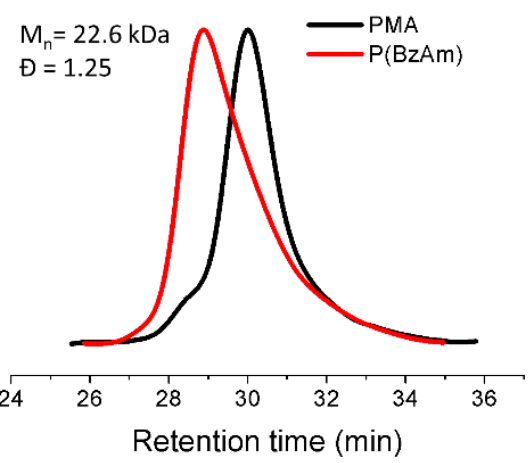

D

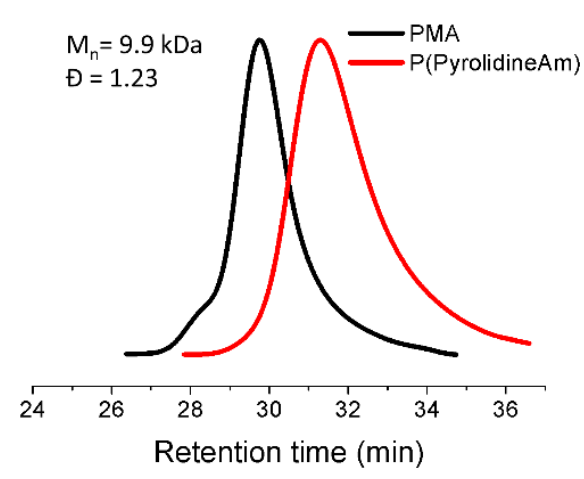

$\mathrm{F}$

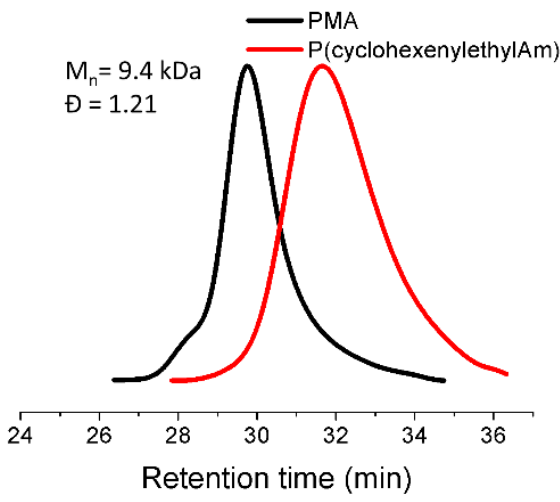

Figure S14: A) Annotated ${ }^{1} H$ NMR spectrum of $\left.P(B z A m)\right)$, B) $P$ (pyrrolidineAm) and C) $P($ cyclohexenylethylAm). B) The corresponding RI-trace in SEC, in red the final polymer and in black the starting material for $P(B z A m)($ top $), D) P($ pyrrolidineAm) and F) $P$ (cyclohexenylethylAm). Mn and $Ð$ are given for the resulting polymers. 


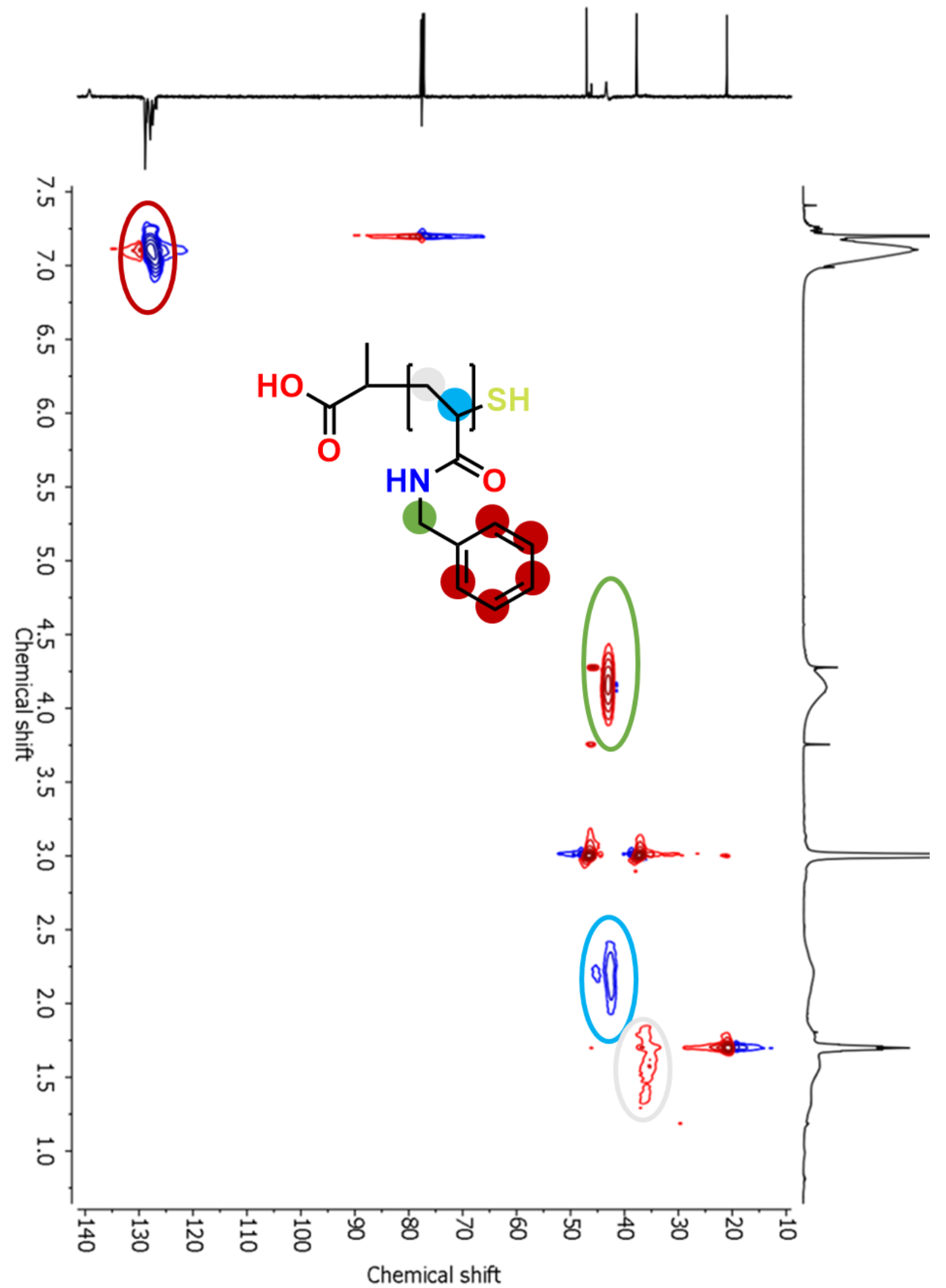

Figure S15: HSQC spectrum of $P(B z A m)$ after full conversion. 


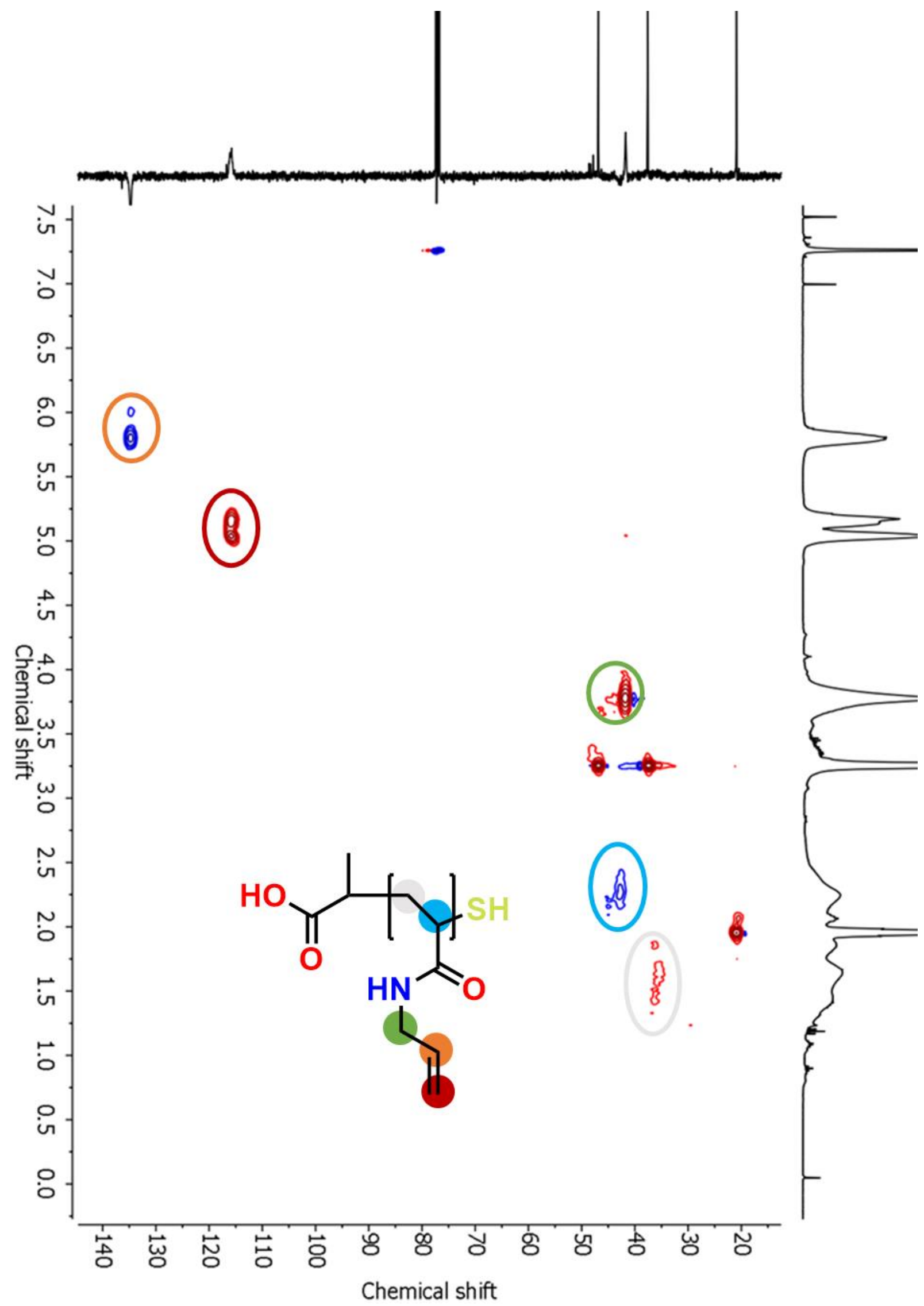

Figure S16: HSQC spectrum of $P(A l l y \mid A m)$ after full conversion. 


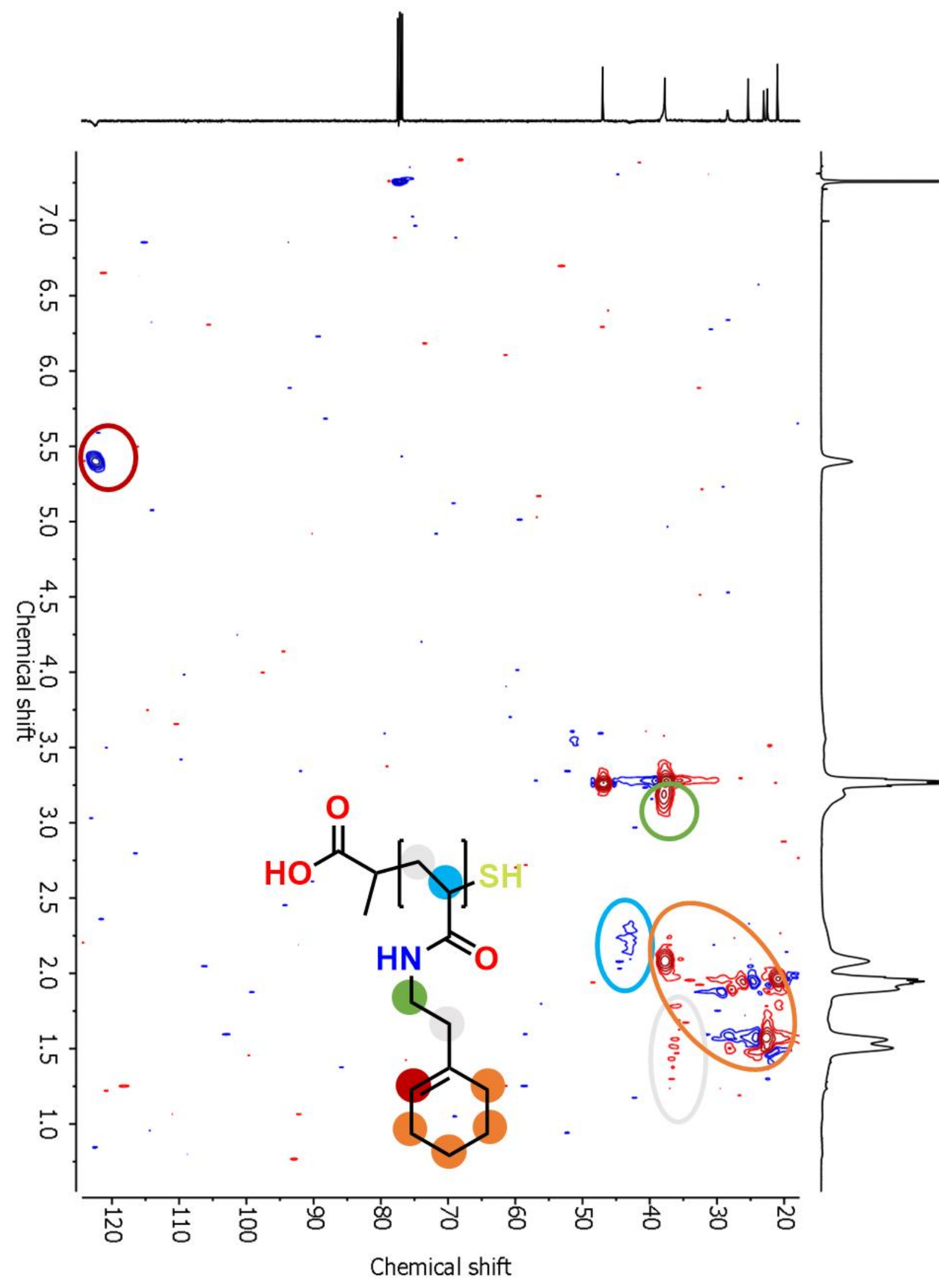

Figure S17 HSQC spectrum of $P($ cyclohexenylethylAm) the final sample. 


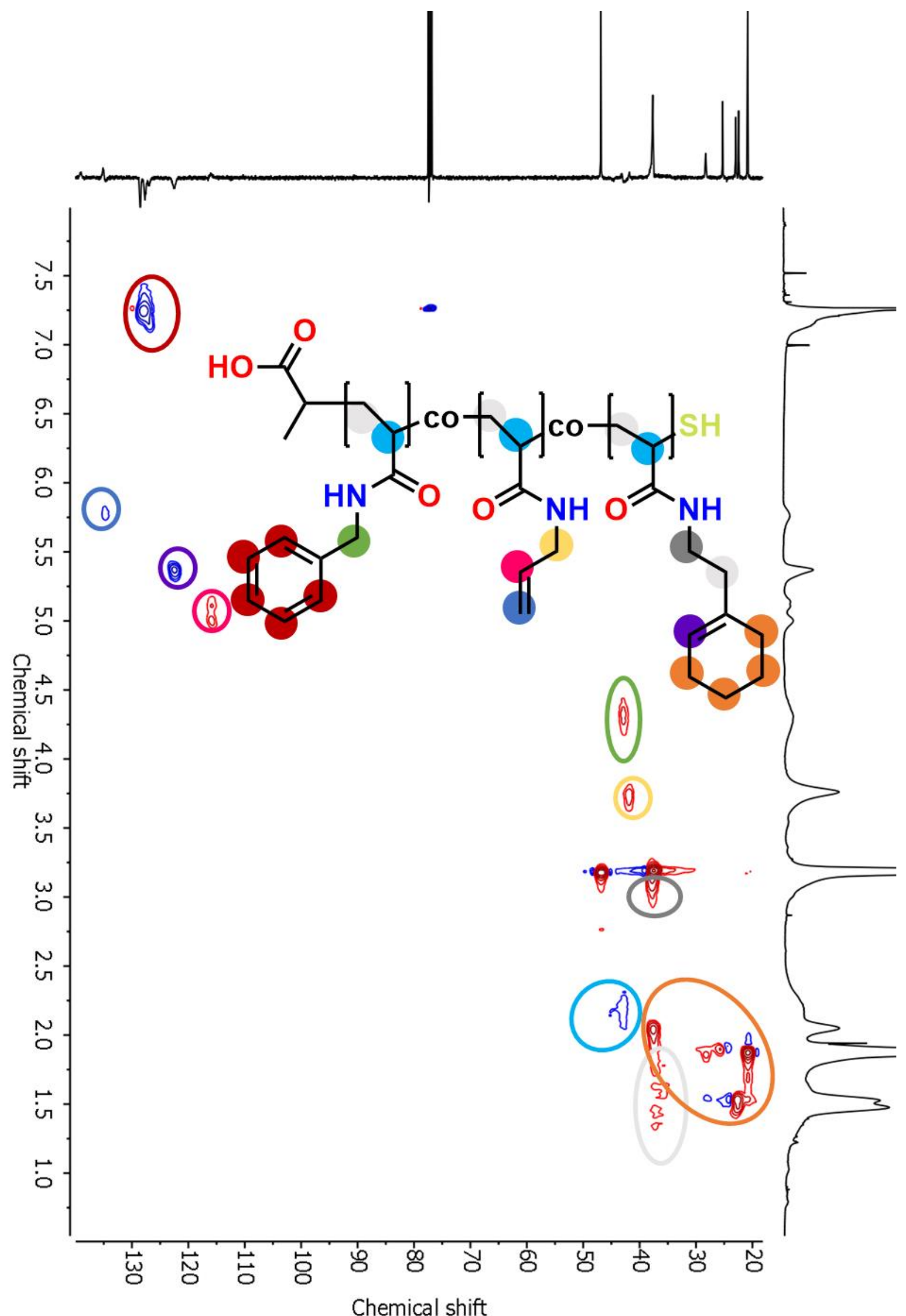

Figure S18: HSQC spectrum of $P(B z A m-c o-A l l y I A m-c o-c y c l o h e x e n y l e t h y l A m)$ after full conversion. 


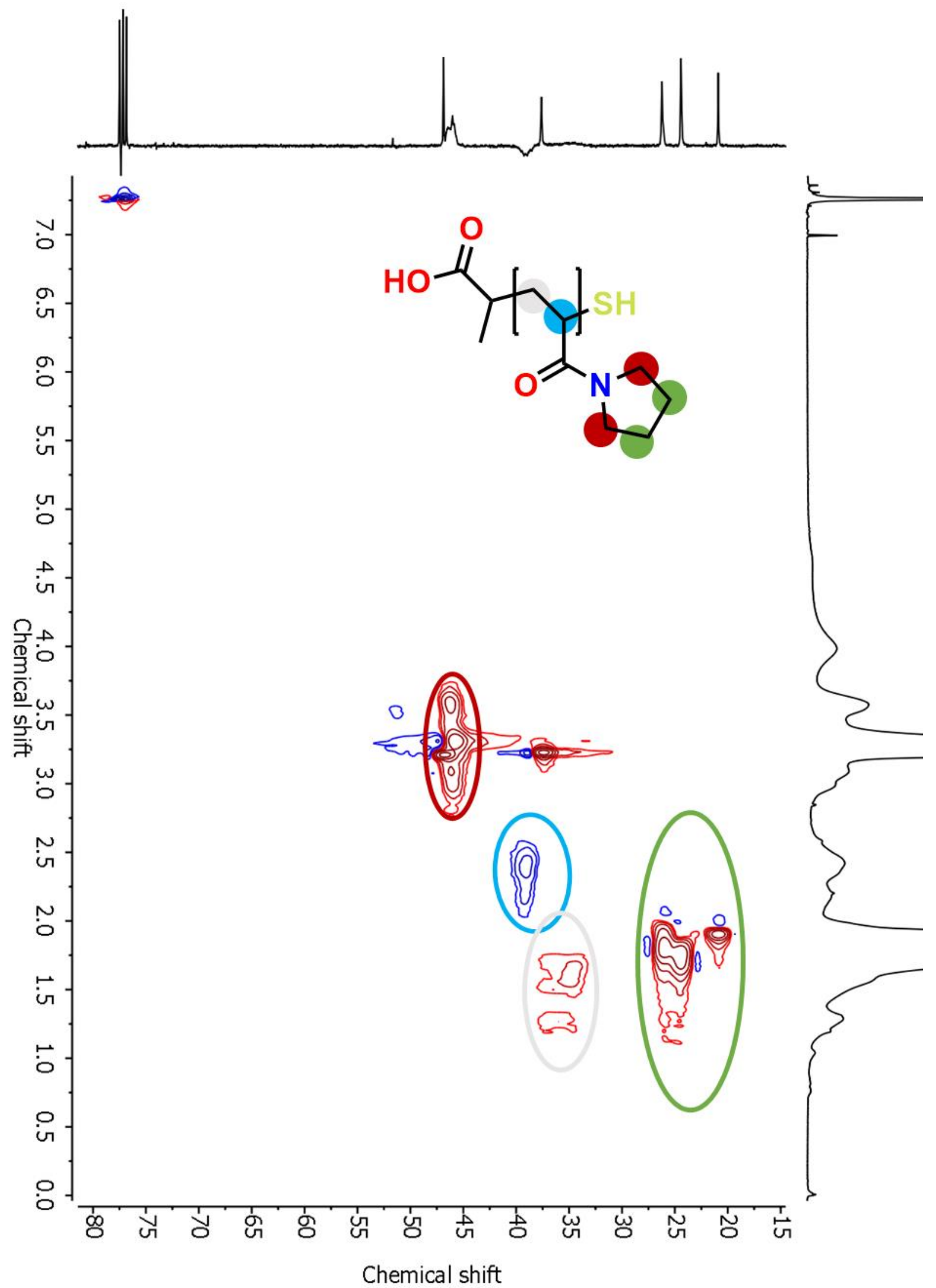

Figure S19: HSQC spectrum of $P($ pyrrolidineAm), the final sample. 


\section{References}

(1) Hall, H. K. Correlation of the Base Strengths of Amines. J. Am. Chem. Soc. , 1957, 79, 5441-5444

(2) Braude, E. A. \& Nachod, F. C. Determination of organic structures by physical methods. Academic Press, New York, 1955) 